\title{
Magnetization reversal with variation of the ratio of the anisotropy energies in exchange bias systems
}

\author{
Amitesh Paul,* Emmanuel Kentzinger, Ulrich Rücker, and Thomas Brückel \\ Institut für Festkörperforschung, Forschungszentrum Jülich GmbH, D-52425 Jülich, Germany
}

(Received 19 June 2006; published 21 August 2006)

\begin{abstract}
We systematically vary the ratio of the exchange and ferromagnetic anisotropies in a single multilayered system in finally unravelling the mysteries of magnetization reversal of exchange coupled systems. This is particularly possible due to increasing unidirectional exchange anisotropies in our multilayer system which we saw earlier from sequential switching of exchange coupled layers along the stack with increasing applied field strengths. Here, by introducing different directions $(\theta)$ of the applied field with respect to the unidirectional anisotropy direction, we have varied two different energy parameters: (i) the exchange anisotropy for each layer, (ii) the ferromagnetic anisotropy. Our polarized neutron measurements thus show a gradual crossover from layer flipping (domain-wall motion) for low torque regimes of the effective field to coherent rotation for high torque regimes. We explain these findings within a general and simple model where the angular dependencies for reversal are guided by the relative strengths of Zeeman, exchange, and anisotropy energies.
\end{abstract}

DOI: 10.1103/PhysRevB.74.054424

PACS number(s): 75.70.Cn, 61.12.Ha, 75.60.Jk

\section{INTRODUCTION}

When a bilayer system of ferromagnet (FM) layer in direct contact with an antiferromagnet $(\mathrm{AF})$ layer is cooled below the Néel temperature a shift in the hysteresis loop is observed. ${ }^{1}$ This shift is given by an exchange bias field which is crucial for designing thin-film magnetic sensors. The magnetization reversal in such systems is complex and is subject to recent studies. Asymmetric hysteresis loops due to different magnetization reversal processes in different branches of the hysteresis loop are common ${ }^{2-7}$ in exchange coupled FM/AF layers. Theoretically the interpretation of the magnetization reversal was discussed in Ref. 8. This is governed by an effective field $H_{\text {eff }}$ arising from the anisotropy of the FM, the exchange bias field of the AF, and the applied field $H_{a} . H_{\text {eff }}$ and the torque it exerts on the FM magnetization depend on the angle $\theta$ between $H_{a}$ and the AF anisotropy axis or the field-cooling axis $H_{\mathrm{FC}}$. Beckmann et al. showed that depending on $\theta$ the reversal mode is either by coherent rotation (uniform reversal mode) for both loop branches or asymmetric with a nonuniform reversal for the increasing branch. Here nonuniform refers to the reversal of magnetization with no component perpendicular to the $H_{a}$ direction which is essentially reversal by domain-wall motion. However, a systematic study of the reversal process by varying the relative strengths of the anisotropy energies which would unravel every observed aspect by various groups on various systems ${ }^{2-7}$ is still lacking and thus has been undertaken here.

Neutron scattering under grazing incidence has proven to be an extremely powerful and unique technique for the investigation of the phenomenon. ${ }^{3-6}$ For one-dimensional analysis four different cross sections can be distinguished, namely non-spin-flip (NSF) $\left(R_{+-+}\right.$and $\left.R_{--}\right)$and spin-flip (SF) channels $\left(R_{+-}\right.$and $\left.R_{-+}\right)$. Here + and - signs to the intensity $R$ represent the polarization parallel and antiparallel to the guiding field. Magnetization rotation is identified by a significant increase of the specular reflectivities in the SF channels, which correspond to in-plane magnetization compo- nents perpendicular to the guiding field $H_{\mathrm{a}}$ applied collinear to $H_{\mathrm{FC}}$. Reversal by domain nucleation and propagation does not provide enhanced SF intensities, because the magnetization is always collinear to $H_{\mathrm{a}}$ and is shown in NSF channels.

Recently we observed sequential reversal of the FM layers in $[\mathrm{IrMn} / \mathrm{CoFe}]_{10},{ }^{9}$ and also in $[\mathrm{Co} / \mathrm{CoO}]_{20}$ multilayers (MLs) ${ }_{10}^{10}$ which was directly related to the evolution of the grain size along the stack which leads to increasing exchange bias strengths along the ML stack. The nonuniform reversal of each CoFe layer was found to proceed symmetrically via domain-wall motion for both remagnetization directions as observed also by imaging techniques. ${ }^{11,12}$ Reversal by coherent magnetization rotation could be excluded because no corresponding specular SF intensity was observed. This reversal mode-symmetric, but nonuniform - corresponds to the situation for $\theta=0^{\circ}$ for our polycrystalline ML specimens, unlike usually observed asymmetric reversals in epitaxial bilayer specimens. ${ }^{4-6,10}$

In the present paper, we investigate the same polycrystalline $\mathrm{IrMn} / \mathrm{CoFe}$ sample. We choose such a prototypical system which shows magnetization reversal that can be symmetric or asymmetric via the nonuniform ${ }^{11}$ or uniform ${ }^{13}$ mode which intuitively may depend upon the respective anisotropy energies involved. Our single multilayered system has different strengths of the bias fields along the ML stack, thus an inherent variation of relative strengths of ferromagnetic and exchange anisotropies is already introduced. This relative strength is predicted to be responsible for reversal of magnetization via coherent rotation or domain-wall motion. Here, by introducing different $\theta$, we have varied two different energy parameters: (i) the exchange anisotropy for each layer, (ii) the ferromagnetic anisotropy. Thus the multidimensional aspect of the anisotropy energy ratio has been used to explain all the facets of magnetization reversal within a single system which obviously do not involve variation of any other unwanted parameters as thickness, preparation conditions, etc. We vary the anisotropy ratio for each layer as we keep the applied field and angle constant while measuring each spectra of specular and off-specular polarized neutron 
scattering from the specimen. We continue this along each full magnetization loop: increasing (negative to positive) and decreasing (positive to negative) field and also varying the sweeping directions to $\theta=90^{\circ}, 45^{\circ}$, and $15^{\circ}$. Grazing incidence polarized neutron scattering is a depth resolved probe which allows us to determine with formidable accuracy the mean magnetization (specular intensity) and its fluctuations (off-specular diffuse scattering) for each layer in the stack. For $\theta=15^{\circ}$, we observe a crossover from sudden flipping of each layer magnetization to coherently rotating layers along the stack for both loop branches. However, for $\theta=45^{\circ}$ and $90^{\circ}$ the reversal proceeds symmetrically and coherently in a uniform mode. The reversal is accompanied by fluctuations of the in-plane magnetization component perpendicular to $H_{\mathrm{a}}$ as observed earlier ${ }^{9,14}$ irrespective of $\theta$.

\section{EXPERIMENT}

The exchange-biased polycrystalline $\left[\operatorname{Ir}_{20} \mathrm{Mn}_{80}(6.0 \mathrm{~nm}) /\right.$ $\left.\mathrm{Co}_{80} \mathrm{Fe}_{20}(3.0 \mathrm{~nm})\right]_{N=10}$ ML is prepared by dc magnetron sputtering. We have performed polarized neutron reflectivity (PNR) measurements at the polarized neutron reflectometer with polarization analysis HADAS at the Jülich research reactor FRJ-2 (DIDO). The details of the instrumental and experimental conditions and microstructural investigations on the specimen were reported previously. ${ }^{9}$ Magnetization loops at different values of $\theta$ were obtained by means of the magneto-optic Kerr effect (MOKE).

\section{RESULTS AND DISCUSSION}

Figures 1(a)-1(d) show longitudinal MOKE magnetization loops for $\theta=90^{\circ}, 45^{\circ}$, and $15^{\circ}$. The loop shape evolves from straight to slanted. This is due to the diminishing magnetization component which is $\|$ to the $H_{a}$ direction.

We performed PNR measurements on both sides of the hysteresis loop at different fields applied for each different $\theta$. We show the measurements with $\theta=15^{\circ}$ for increasing and decreasing fields in Fig. 2. The specular intensity along the line $\alpha_{\mathrm{i}}=\alpha_{\mathrm{f}}$ shows first-order and weaker second-order Bragg peaks at $\alpha_{\mathrm{i}, \mathrm{f}} \approx 25$ and $50 \mathrm{mrad}$, respectively, corresponding to the bilayer thickness. The variation of the SF intensity peaks with $H_{a}$ (at the critical angle of total reflection) with respect to that in the saturation state indicate the correlation of the magnetization evolution along the stack of the ML.

The fitting of the specular reflectivities for the NSF and SF channels are done taking into account the nonideal polarization efficiencies. The details of the fitting procedure have been described earlier. ${ }^{9}$ In Fig. 3 we show the variation of the angle of magnetization $\phi_{A}$ extracted from the fits to the specular data. When the magnetization is parallel to the applied field $\phi_{A}=0$, thus an increase in $\phi_{A}$ is visible by an increase in the SF signal. For $\theta=90^{\circ}$ and $45^{\circ}$, we observe monotonically varying large specular SF signal all along the hysteresis loop, indicating symmetric, uniform, and simultaneous magnetization reversal of all layers by coherent rotation. ${ }^{14}$ For $\theta=15^{\circ}$, each layer rotates or flips independently and sequentially with field strength, fanning around in different directions (see layer schematic) in a certain field

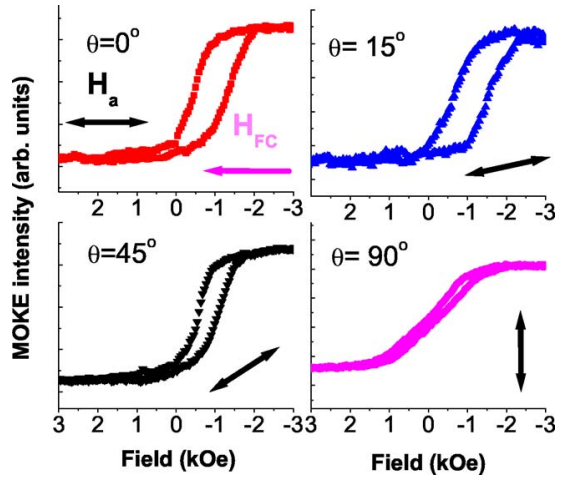

FIG. 1. (Color online) MOKE hysteresis loops for $\left[\operatorname{Ir}_{20} \mathrm{Mn}_{80}(6.0 \mathrm{~nm}) / \mathrm{Co}_{80} \mathrm{Fe}_{20}(3.0 \mathrm{~nm})\right]_{10}$ ML with $\theta=0^{\circ}, 15^{\circ}, 45^{\circ}$, and $90^{\circ}$. Here $\theta$ is the angle between the $H_{F C}$ and $H_{a}$ directions.

which shows the layer resolved magnetization direction. On the deceasing field branch: the bottom layers start flipping first followed by the rotating middle and again flipping top layers as we increase the field strength. On the increasing branch: the top layers flip back while the middle layers rotate in different directions and the very bottom layers flip and rotate back. The flipping and rotation of layers are seen by the presence or absence of SF signals during field sweeping. We explain the above observations assuming a simple model where the relevant energy terms can be written as

$$
E=-H_{a} M_{F M} \cos (\delta-\theta)-J M_{F M} M_{A F} \cos \delta+k \sin ^{2} \delta,
$$

where $J$ is the interlayer exchange strength between FM and AF layers. Here, $\delta$ is the angle between the $M_{F M}$ and the easy axis. $M_{F M}, M_{A F}$ (uncompensated spins) are the saturation magnetization of the FM and magnetization of the $\mathrm{AF}$ layer, $k$ is the uniaxial FM anisotropy and $H_{X}\left(=J M_{A F}\right)$ is the exchange field. Here we consider $M_{A F}$ along the easy axis which is assumed to be parallel to the $H_{F C}$ direction and furthermore assume that $M_{A F}$ does not rotate with the $H_{a}$ direction $^{15}$ and also that the domain-wall energy is large compared to the interfacial exchange energy. ${ }^{16}$

The regimes of different anisotropy energies involved here are shown in Fig. 4 which is varying along the stack as well as with $\theta$. The exchange anisotropy is given by $J_{X}$ $=M_{F M} H_{X}$. We estimate $k\left(=H_{S} M_{F M} / 2\right)$ from the fit to the MOKE hysteresis loops at $\theta=90^{\circ}\left(H_{S}\right.$ is the saturation field) and $H_{X}$ from the loop shift at different $\theta$ of the experimental data for samples with different $N$. Intrinsically, each layer in the stack has different $k$ and different $H_{X}$ values, which causes change in the ratio of $k(N) / J_{X}(N)$. Further, for each $k$ we introduce different $J_{X}$ as we change $\theta$, thereby varying the ratio as $k(N) / J_{X}(N, \theta)$.

For a finite $\theta$, the strength of the anisotropy field $H_{A}$ (depend on the projection of the FM magnetization onto the easy axis) and that of the exchange field $\left(H_{X}\right)$ decide on the angle $\left(\phi_{1}\right)$ between the effective field $H_{\text {eff }}\left(H_{\text {eff }}=H_{A}+H_{a}\right.$ $+H_{X}$ ) and the $M_{F M}$ direction at equilibrium for the increasing branch of the hysteresis loop. As the sign of the FM magnetization (decreasing branch of the hysteresis loop) reverses so 


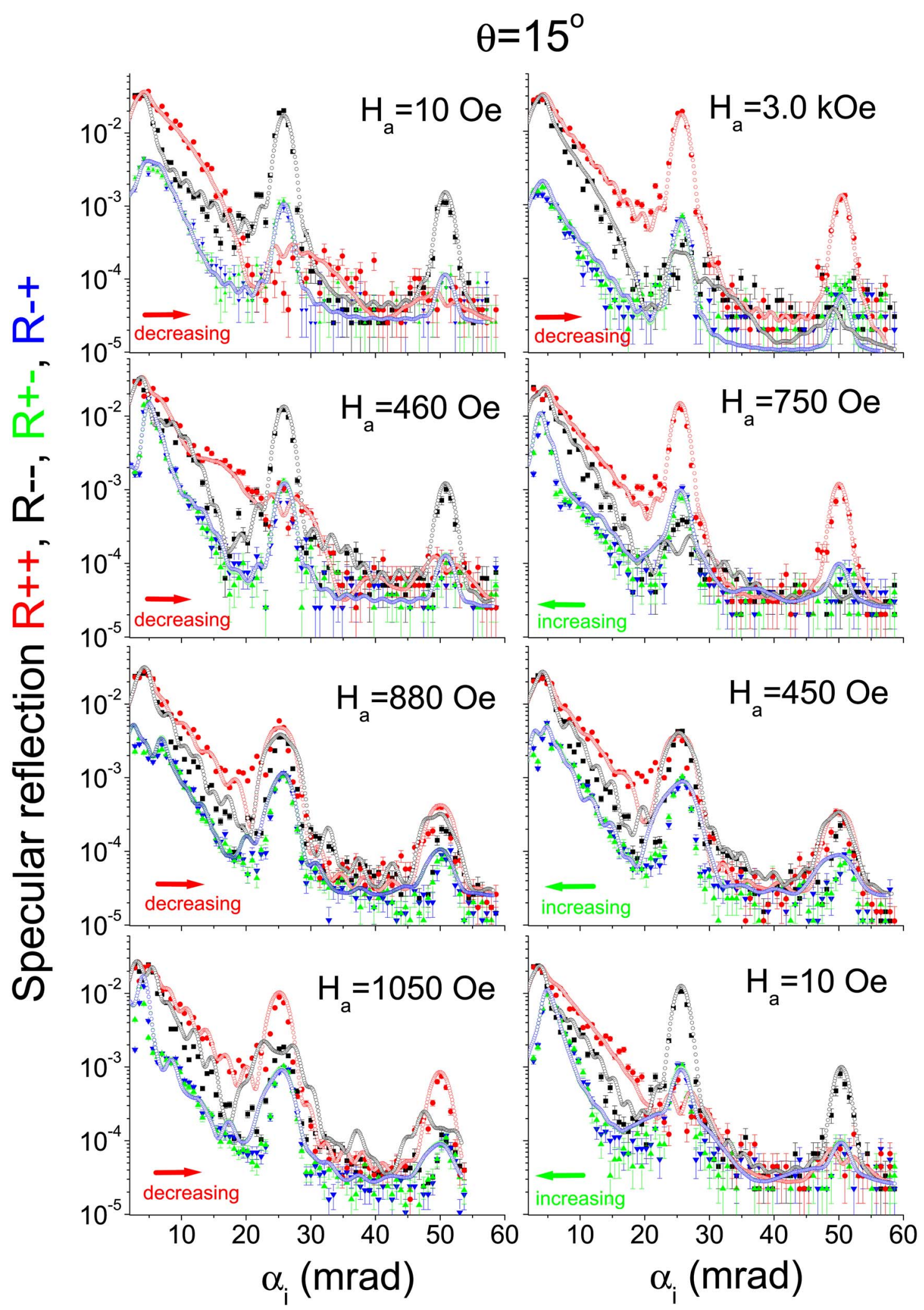

FIG. 2. (Color online) NSF $\left[R_{--}\right.$(black) squares and $R_{++}$(red/dark gray) circles $]$and SF $\left[R_{+-}\right.$(green/light gray) triangles and $R_{-+}$ (blue/black) downtriangles] specular reflectivity patterns along with their fits (open circles) for $\left[\operatorname{Ir}_{20} \mathrm{Mn}_{80}(6.0 \mathrm{~nm}) / \mathrm{Co}_{80} \mathrm{Fe}_{20}(3.0 \mathrm{~nm})\right]_{10} \mathrm{ML}$ with $\theta=15^{\circ}$ for at representative fields along the increasing and decreasing branches of the field. The SF signals at different fields can be compared with that of the saturation state at $3.0 \mathrm{kOe}$ where the signal in the SF channels are due to nonideal polarization only. 


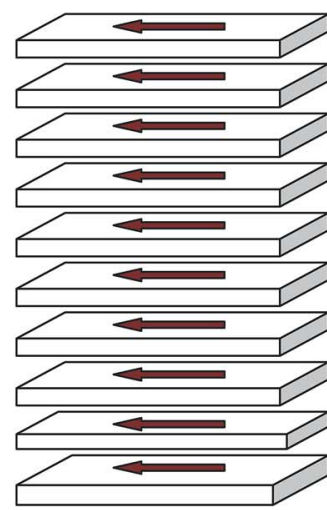

$0.0 \mathrm{Oe}$

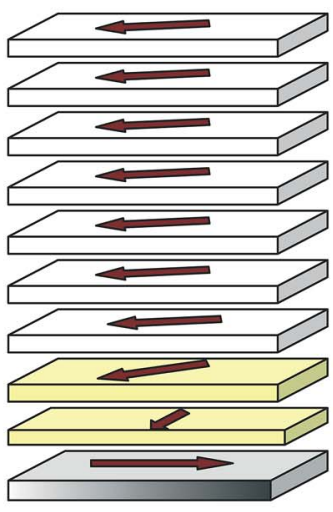

$0.5 \mathrm{kOe}$

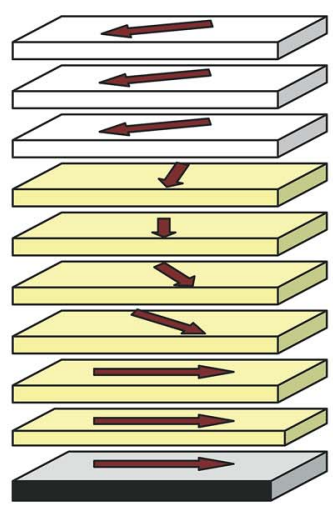

$1.0 \mathrm{kOe}$

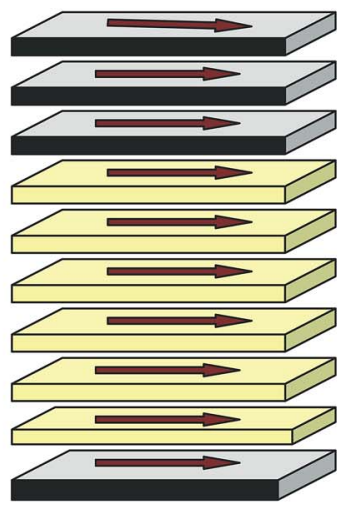

$4.0 \mathrm{kOe}$

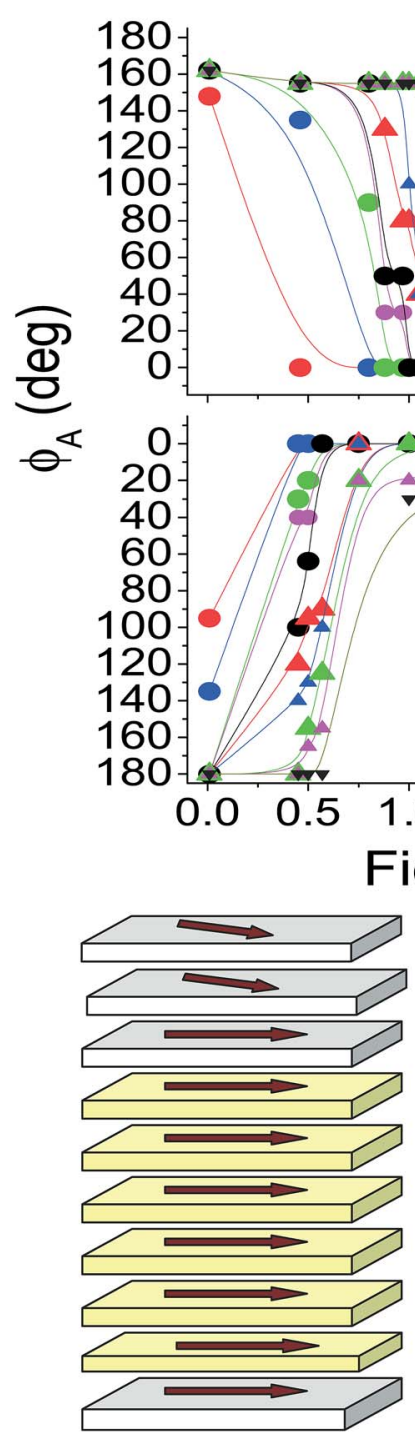

$1.0 \mathrm{kOe}$

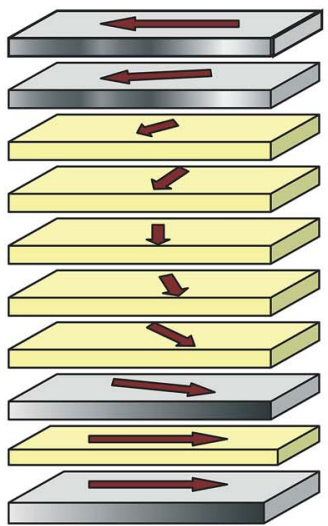

$0.5 \mathrm{kOe}$

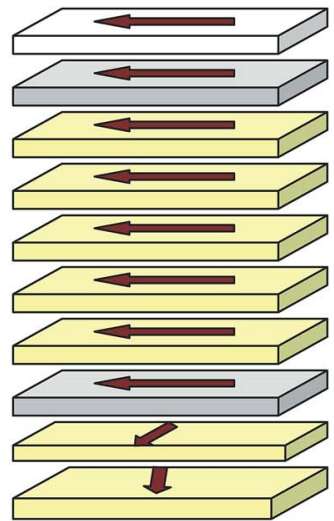

$0.0 \mathrm{Oe}$

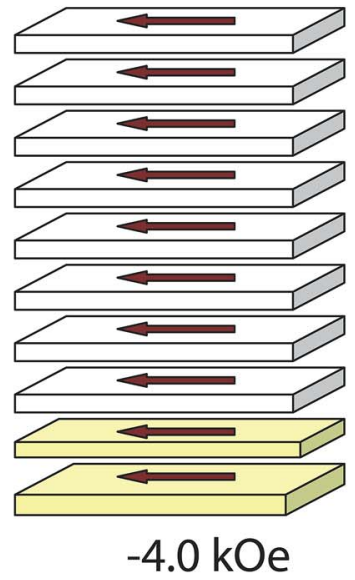

FIG. 3. (Color online) Variation of the angle of magnetization $\phi_{A}$ for $\left[\operatorname{Ir}_{20} \mathrm{Mn}_{80}(6.0 \mathrm{~nm}) / \mathrm{Co}_{80} \mathrm{Fe}_{20}(3.0 \mathrm{~nm})\right]_{10} \mathrm{ML}$ with $\theta=15^{\circ}$ for increasing and decreasing fields as obtained from the fits of the specular reflectivity patterns. The lines are guide to the eye and the size of the symbols are the error bars. A schematic of the reversal for each layer is also shown where we find separately rotating (yellow/light gray) and flipping (shaded gray) layers as compared to that of collective rotation of all layers when $\theta=45^{\circ} / 90^{\circ}$ (Ref. 14). Inset shows the scattering geometry. 


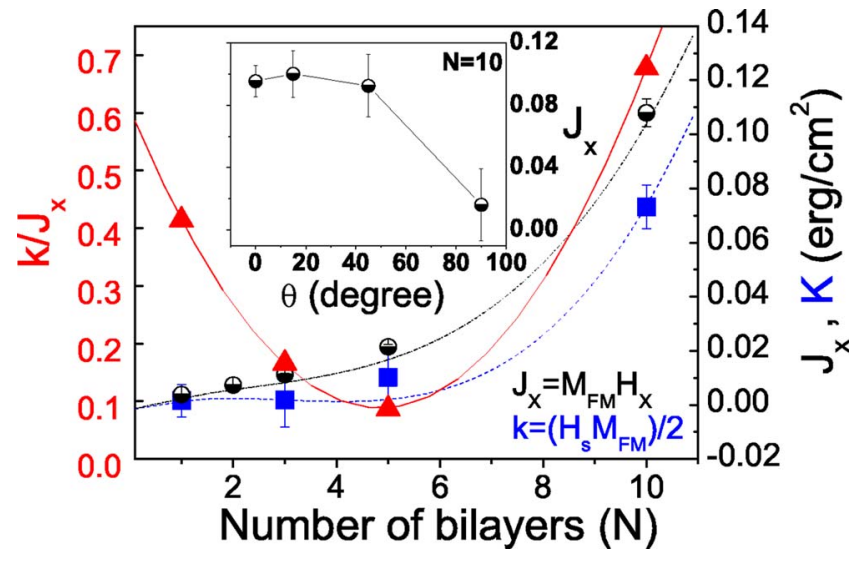

FIG. 4. (Color online) Plot of the range of anisotropy energies (i) across the ML stack and also (ii) as we vary the applied field angles (inset) for $N=10$ bilayers as obtained from MOKE curves: thus changing the ratio (red/gray triangles) of exchange $\left(J_{X}\right)$ (black half circles) and uniaxial ( $k$ ) anisotropies (blue/black squares). The lines are to guide the eye.

does the anisotropy field and the corresponding angle $\phi_{2}$. Larger angle means larger torque which favors rotation of the magnetization: this is the case for our system when $\theta=90^{\circ}$ and $45^{\circ}$, where we see symmetric and simultaneous rotation of all the layers for increasing and decreasing branch which are otherwise decoupled. ${ }^{14}$ Whereas a small angle favors flipping by domain-wall motion. ${ }^{9,10}$

Comparing the anisotropy ratio in Fig. 4 for $\theta \leqslant 15^{\circ}$, one can see that it is significant for the bottom layer magntization which flips with $H_{a}$. In case of $H_{X} \neq H_{A}$ and also where the ratio of anisotropies is minimum, one expects an increase in $\phi_{1,2}$. Therefore we generally observe magnetization rotation for the middle layers. A sketch showing the angle $H_{\text {eff }}$ makes with the $M_{F M}$ for representative strengths of the anisotropy and exchange field is presented in Fig. 5(a). However, when $H_{X} \approx H_{A}$, one may have asymmetric reversal as nonuniform reversal may occur for the increasing branch (alignment of $H_{e f f}$ with $M_{F M}: \phi_{1} \sim 0^{\circ}$ )—possibly even for $\theta>0^{\circ},{ }^{14}$ as also shown by Monte Carlo simulation. ${ }^{8}$ In the case of large anisotropy ratio, one can also expect asymmetric reversal (nonuniform reversal for the decreasing branch) as shown for $\theta \simeq 0^{\circ} .{ }^{10}$ With further increase in $H_{a}$, we approach even higher values of $H_{X}$ thereby increasing the anisotropy ratio again. This causes the $\phi_{1,2}$ to approach $0^{\circ} / 180^{\circ}$ which favors flipping of the top layers [Fig. 5(b)]. For our system, for

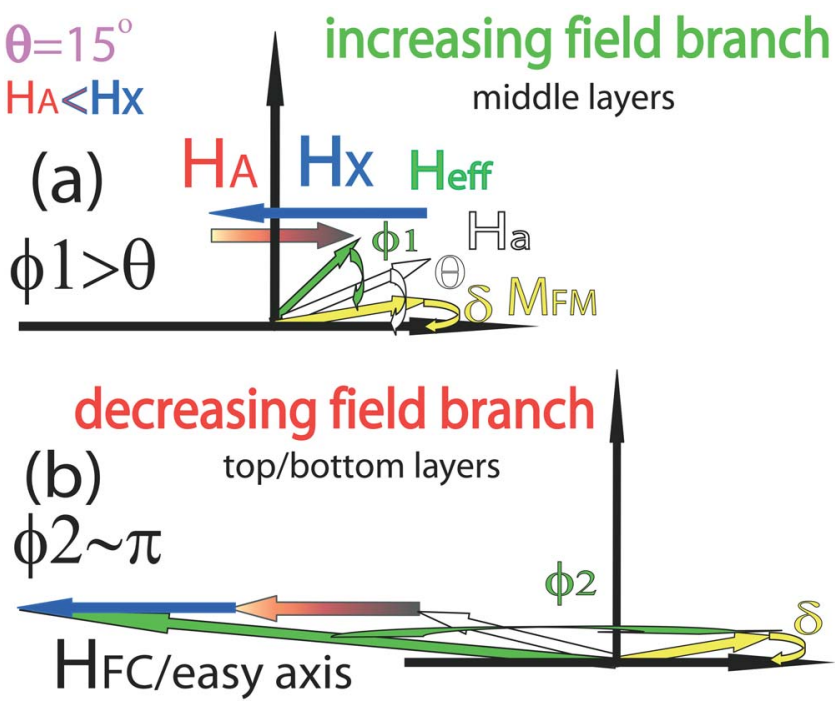

FIG. 5. (Color online) Sketch showing the $H_{\text {eff }}$ (green/gray) along the hysteresis loop of the ML: (a) increasing branch for middle layers, and (b) decreasing branch for top layers for $\theta \approx 15^{\circ}$ depicting situations when $H_{X}$ (blue/black) $\gg H_{A}$ (shaded red/gray). The $M_{F M}$ (yellow/light gray) and $H_{a}$ (white) are making an angle $\delta$ and $\theta$, respectively, with the easy axis which is the $H_{F C}$ direction. The components of the fields $\perp$ to $H_{F C}$ has been exaggerated.

$\theta \approx 15^{\circ}$, we observe therefore the crossover from magnetization rotation to magnetization flipping correlated to the systematic variation of anisotropy energies in individual layers.

\section{CONCLUSION}

In conclusion, we systematically varied the relative strengths of the exchange and ferromagnetic anisotropy energies in a single multilayered system which is particularly possible due to sequential switching of layers with increasing layer numbers. Remagnetization behavior of such an exchange-biased multilayer (varied along the stack) for different directions $\theta$ (another variable parameter) let us observe layer resolved symmetric reversal via nonuniform or uniform modes and asymmetric reversal via nonuniform and uniform modes. These observations are understood in general terms of the torque exerted on the system due to different effective field angles with respect to the magnetization axis. Our measurements show a gradual crossover from layer flipping (domain-wall motion) for low torque regimes to coherent rotation for high torque regimes. Our experimental findings finally unravel the mysteries of magnetization reversal of exchange coupled systems and explain all earlier observations of asymmetric and symmetric hysteresis loops.
*Author to whom correspondence should be addressed. Electronic address: A.Paul@fz-juelich.de

${ }^{1}$ W. H. Meiklejohn and C. P. Bean, Phys. Rev. 102, 1413 (1956).

${ }^{2}$ V. I. Nikitenko, V. S. Gornakov, A. J. Shapiro, R. D. Shull, Kai Liu, S. M. Zhou, and C. L. Chien, Phys. Rev. Lett. 84, 765
(2000).

${ }^{3}$ W.-T. Lee, S. G. E. te Velthuis, G. P. Felcher, F. Klose, T. Gredig, and E. D. Dahlberg, Phys. Rev. B 65, 224417 (2002).

${ }^{4}$ M. R. Fitzsimmons, P. Yashar, C. Leighton, Ivan K. Schuller, J. Nogues, C. F. Majkrzak, and J. A. Dura, Phys. Rev. Lett. 84, 
3986 (2000).

${ }^{5}$ M. Gierlings, M. J. Prandolini, H. Fritzsche, M. Gruyters, and D. Riegel, Phys. Rev. B 65, 092407 (2002).

${ }^{6}$ F. Radu, M. Etzkorn, R. Siebrecht, T. Schmitte, K. Westerholt, and H. Zabel, Phys. Rev. B 67, 134409 (2003).

${ }^{7}$ E. Arenholz and K. Liu, Appl. Phys. Lett. 87, 132501 (2005).

${ }^{8}$ B. Beckmann, U. Nowak, and K. D. Usadel, Phys. Rev. Lett. 91, 187201 (2003).

${ }^{9}$ A. Paul, D. E. Bürgler, and P. Grünberg, J. Magn. Magn. Mater. 286, 216 (2005); A. Paul, E. Kentzinger, U. Rücker, D. E. Bürgler, and P. Grünberg, Phys. Rev. B 70, 224410 (2004).

${ }^{10}$ A. Paul, E. Kentzinger, U. Rücker, and Th. Brückel, Phys. Rev. B 73, 092410 (2006).

${ }^{11}$ P. Gogol, J. N. Chapman, M. F. Gillies, and F. W. M. Vanhelmont,
J. Appl. Phys. 92, 1458 (2002).

${ }^{12}$ A. Kirilyuk, Th. Rasing, H. Jaffrès, D. Lacour, and F. Nguyen Van Dau, J. Appl. Phys. 91, 7745 (2002).

${ }^{13}$ Florin Radu, Andreas Westphalen, Katharina Theis-Bröhl, and Hartmut Zabel, J. Phys.: Condens. Matter 18, L29 (2006).

${ }^{14}$ A. Paul, E. Kentzinger, U. Rücker, and Th. Brückel, J. Phys.: Condens. Matter 18, L149 (2006); J. Appl. Phys. (to be published).

${ }^{15}$ H. Ohldag, A. Scholl, F. Nolting, E. Arenholz, S. Maat, A. T. Young, M. Carey, and J. Stohr, Phys. Rev. Lett. 91, 017203 (2003).

${ }^{16}$ A. E. Berkowitz and K. Takano, J. Magn. Magn. Mater. 200, 522 (1999). 